\title{
Eventización: los acontecimientos mediáticos como estrategia pública de legitimación y posicionamiento en la red global de ciudades turísticas
}

Javier Hernández-Ramírez. Universidad de Sevilla, Sevilla, España.

Auxiliadora Comendador-Sánchez. Universidad de Sevilla, Sevilla, España.

RESUMEN | En este artículo se aborda la apuesta decidida del gobierno municipal de Sevilla (Andalucía, España) por programar un calendario ininterrumpido de grandes acontecimientos mediáticos que alcanzan un importante impacto internacional. A través del análisis de los discursos de responsables públicos y privados, en el estudio se constata que esta política municipal de internacionalización y singularización constituye la piedra angular de la estrategia turística, funciona como un eficaz instrumento de legitimación y es una vía para la inmersión de la economía local en la lógica global.

PALABRAS CLAVE | competitividad urbana, gobierno local, política urbana.

ABSTRACT | This study addresses the determined commitment of the municipal government of Seville (Andalusia, Spain) to schedule an uninterrupted calendar of major media events, with the purpose of achieving significant international impact. Through the analysis of the discourse of public and private decision makers, the study confirms that the municipal policy of internationalization and singularization, constitutes the cornerstone of a strategy for tourism, functions as an effective instrument of legitimation, and is a means by which the local economy is immersed in the global logic.

KEYWORDS | urban competitiveness, local government, urban policy. 


\section{Introducción}

Desde el último cuarto del siglo xx, son muchas las ciudades que han pasado de ser centros manufactureros, a constituirse en focos financieros y espacios de creación, consumo y entretenimiento. Esta evolución de lugar de producción a espacio y objeto de consumo ha exigido una profunda metamorfosis y la puesta en marcha de una serie de iniciativas de internacionalización. Entre ellas destacan la comercialización de la urbe como un producto atractivo e identificable ("city branding"), la inversión en equipamientos e infraestructuras de accesibilidad y conectividad y la ejecución de todo un conjunto de actuaciones urbanísticas que persiguen generar productos culturales diversificados, capaces de seducir a consumidores cosmopolitas.

La última iniciativa es la de mayor calado. Entre las intervenciones que ha generado, destacan una política patrimonial que estetiza la ciudad, especialmente el recinto histórico ("museificación"); la erección de nuevos edificios emblemáticos, que deslumbran por su innovación, osadía arquitectónica o gigantismo ("neomonumentalismo"); la celebración de grandes eventos internacionales que alcanzan una espectacular proyección internacional ("megaeventos"); y, por último, la organización de cumbres, efemérides y acontecimientos mediáticos que transforman la ciudad en escenario de encuentros internacionales de lo más variopinto ("eventización”). Paradójicamente, estas iniciativas de singularización urbana se reproducen por doquier, generándose un modelo de ciudad que, por su reiteración, es casi clónico (Muñoz, 2008). De este modo el turismo forja una impronta urbana global replicada, que es característica de la actual modernidad tardía.

Las actuaciones señaladas son abordadas especialmente en aquellas urbes cuyos grupos políticos y empresariales aspiran a convertirlas en nodos turísticos globales. Para ello pugnan por posicionarse ventajosamente en una jerarquizada y policéntrica red de ciudades dentro de un inestable marco de competencia global/local. El objetivo de cada aspirante es situarse como un indiscutible destino turístico internacional en la tabla clasificatoria de las ciudades globales. Aquellas urbes que alcanzan éxito por sus actuaciones, y consecuente proyección internacional, consiguen situarse favorablemente en este archipiélago mundial de ciudades privilegiadas (Gago et al., 2017; Pisonero, 2017). No obstante, dada la fuerte competencia existente, este posicionamiento puede ser efímero o coyuntural, por lo que las ciudades deben afrontar un permanente y casi frenético esfuerzo de renovación para mantenerse en el candelero mediático y así atraer tanto los flujos de turistas como las inversiones exteriores.

Esta reorientación de las políticas municipales desde lo local a lo global se produce en el marco de una estrategia neoliberal que persigue la absorción de parte de los beneficios y los flujos derivados de la actual sociedad móvil mundial (Hall, 2006; Salazar, 2016). En este contexto de internacionalización, las ciudades son gestionadas como empresas (Harvey, 1989), por lo que se apuesta por la atracción de capitales y turistas, lo que aparece como justificación de todas las iniciativas mencionadas. El resultado es que el turismo termina siendo una de las fuerzas más determinantes en la producción de ciudad. Pero el riesgo de esta especialización es que los objetivos de captación de capitales y visitantes puedan anteponerse a la 
satisfacción de las necesidades básicas de la población local y la continuidad de sus modos de vida.

El presente artículo toma como unidad de estudio la ciudad de Sevilla (Andalucía, España) y centra su análisis en la iniciativa política de la "eventización", la cual se basa en el diseño, programación y celebración de una sucesión de acontecimientos mediáticos de alcance internacional. Como se mostrará en este trabajo, la eventización constituye la piedra angular de la estrategia turística que el consistorio hispalense ha desarrollado en los últimos años. Con este estudio de caso se pretende contribuir al conocimiento y compresión de la eventización, entendida como una de las actuaciones más relevantes de singularización y posicionamiento urbano en el marco del neoliberalismo. El trabajo se encuadra en la teoría crítica sobre los eventos turísticos ("critical event tourism"), la cual aborda el fenómeno atendiendo a sus contextos sociales, culturales y políticos más amplios (Lamond \& Platt, 2016; Robertson et al., 2018; Spracklen \& Lamond, 2016). En la presente investigación se presta especial atención a la dimensión política de los eventos desde un enfoque local y su aportación principal es que se centra en el análisis de los discursos emitidos por sus principales promotores públicos y privados. Con esta aproximación se pretende alcanzar dos objetivos bien definidos: de un lado, constatar que esta política municipal de internacionalización y singularización persigue la integración plena de la economía local en la lógica global; y, de otro, enfatizar que existe una narrativa perfectamente estudiada dirigida a buscar el respaldo social a la política eventizadora, que funciona además como un eficaz instrumento de legitimación. Esta perspectiva crítica pretende problematizar otras aproximaciones más habituales que abordan la eventización a partir del análisis de sus beneficios económicos, sociales, urbanísticos y de imagen (Ganau, 2007; Getz, 2013; Herrero et al., 2006; Paül i Agustí, 2013; Richards \& Colombo, 2017; Richards \& Palmer, 2010; Richards \& Ruiz, 2017; Richards \& Wilson, 2004) o analizan su influencia como estrategia de posicionamiento dentro de los rankings urbanos globales (Gago et al., 2017; Pisonero, 2017).

El artículo se estructura en seis apartados, que comienzan con esta introducción. En el segundo se contextualizan y definen los grandes acontecimientos mediáticos como un fenómeno característico de las políticas públicas urbanas, que prolifera a escala planetaria. El trabajo continúa con un tercer apartado centrado en la metodología cualitativa empleada. Posteriormente, se presenta la unidad de estudio y se explica el sentido y los objetivos locales de la estrategia de eventización. El quinto se subdivide en tres epígrafes, donde se presentan los resultados de la investigación derivados del análisis de contenido y semiótico aplicado. El artículo concluye con unas consideraciones finales donde se discuten los resultados y se plantean interrogantes y retos para investigaciones futuras.

\section{Marco teórico y contextualización}

Aunque la idea de la ciudad como escenario no es nueva, ya que acompaña al mundo moderno desde el siglo xIx, en la modernidad tardía el fenómeno se ha acentuado y expandido por muchos rincones del planeta. Hoy, las "ciudades festivas" 
no constituyen un reducido y exclusivo elenco (González \& Morales, 2009, 2017; Gravari-Barbas, 2007). Todo lo contrario; desde hace unas pocas décadas aumenta el número de las que aspiran a convertirse en sede de grandes espectáculos, conmemoraciones y cumbres internacionales, en una muy competitiva pugna global (Escudero, 2019). El fenómeno revela que la apuesta por la eventización caracteriza las políticas públicas de las ciudades que desean posicionarse ventajosamente en la red de urbes globales (Pisonero, 2017). De hecho, muchas compiten como candidatas para ser la sede de citas de resonancia internacional, copiando modelos de éxito e incluso "robando" eventos de sus vecinas (Brito \& Richards, 2017; González, 2011; Van Aalst \& van Melik, 2012). ${ }^{1}$

Podría decirse que estos acontecimientos son una de las materializaciones más claras de la sociedad del espectáculo (Debord, 1992). Se enmarcan en las tendencias hacia la "festivalización" de las ciudades y la "eventización" de la cultura (Cudny, 2016; Gravari-Barbas, 2007; Négrier, 2011; Richards \& Palmer, 2010; Spracklen et al., 2013). Ambos procesos contemporáneos se traducen en la proliferación de festivales que son del todo ajenos al sentido de las fiestas tradicionales como lugares de expresión colectiva y ritual, pues se inscriben en el orden y las lógicas del consumo (internacionalización, espectáculo, movilidad, inmediatez, estetización, hedonismo, individualización) (Lipovetski \& Seroy, 2015; Simonicca, 1997). De cara al exterior, el objetivo principal es atraer visitantes e inversores, pero también singularizar la imagen internacional del lugar donde se celebran, razón por la cual algunos autores los denominan "hallmark events", "special events" o "eventos distintivos" (Escudero, 2019; Getz, 2012; Jago \& Shaw, 1998; Ritchie \& Beliveau, 1974). De cara al consumo interno, estas actuaciones son promocionadas como un medio idóneo para impulsar el crecimiento no solo turístico sino de la economía en general, lo cual genera consensos y réditos electorales nada despreciables que, en ocasiones, sirven para pacificar el descontento social y político vinculado a las desigualdades sociales (Shin, 2012).

La celebración de eventos comparte en todos los lugares y situaciones un metaobjetivo: funcionar como una potente actuación promocional. Y esta proyección mediática internacional queda asegurada, porque los acontecimientos en referencia se ofrecen a la visita no solo presencial, sino también virtual de millones de espectadores, gracias a las tecnologías de la comunicación que han hecho posible la existencia de una audiencia a escala planetaria. Como sugiere Hall (2006), la importancia que han alcanzado los grandes encuentros está estrechamente relacionada con la globalización de los medios de comunicación.

Los acontecimientos mediáticos convierten a la ciudad en capital internacional, lo que incide en su imagen y propaga sus atractivos, modelando un tipo específico de urbe contemporánea que es denominada "eventful city" (Richards \& Palmer, 2010). Sin embargo, esta centralidad se produce por un espacio de tiempo determinado, que es generalmente breve. Debido a esta condición fugaz, una vez finalizados los eventos tienen que ser reemplazados por otros nuevos de calado internacional, si

1 Por ejemplo, es notoria la competencia entre Barcelona y Madrid por ser la sede del Mobile World Congress (MwC) (El Pais, 16/01/2020). 
lo que se desea es mantener a la ciudad en el candelero mediático como urbe global, festiva, luminosa y seductora para la visita y la atracción de inversores. La estrategia no deja otra opción, lo que condiciona la orientación de la política pública local. Esta especialización en la celebración sucesiva de eventos de corta duración ha llevado a algunos autores a hablar de "ciudades efímeras" (González \& Morales, 2009). En ellas se impone una política cultural basada en la programación de eventos breves y coyunturales que a menudo son móviles, porque cambian periódicamente de sede, lo cual contrasta con los de épocas anteriores, caracterizados por ser permanentes y celebrados en lugares fijos (Négrier, 2011). Por todo lo anterior puede decirse que las ciudades efímeras y la eventización son la expresión y el paradigma de la cultura de lo eventual y de la desterritorialización de la cultura, fenómenos característicos de la modernidad tardía.

La especialización de la ciudad efímera en una programación sucesiva de acontecimientos de impacto internacional permite la generación de toda una red de conexiones alrededor del mundo, que funciona como uno de los instrumentos fundamentales de la política local para fomentar el crecimiento. Los responsables públicos y privados de las ciudades que se especializan en la producción de eventos en serie compiten para posicionarse ventajosamente en estas redes globales, lo que dependerá de su capacidad para instrumentalizar sus vínculos y así poder establecer una agenda de nuevos eventos de impacto, que permitan mejorar su imagen, captar inversiones y atraer turistas (Richards \& Colombo, 2017).

Otro rasgo que identifica a estos acontecimientos mediáticos es su variabilidad temática, ya que las citas de carácter internacional proyectan a la ciudad desde ámbitos tan distintos como los grandes conciertos, los festivales cinematográficos, los certámenes gastronómicos, las efemérides y conmemoraciones, los campeonatos deportivos, las ferias de productos y actividades, los encuentros sectoriales, los congresos profesionales, las galas de entrega de premios y un largo etcétera. Es decir, acogen tanto espectáculos de masas como eventos más exclusivos y/o corporativos. Entre estos últimos destacan las cumbres internacionales destinadas y reservadas a agentes específicos y especializados (altos directivos, líderes políticos, intelectuales, empresarios, financieros...) que sobresalen por su cualificación, capital humano y/o poder económico. Por el contrario, los espectáculos culturales suelen orientarse a un público internacional más indiferenciado. Estos festivales de masas dejan su huella e imprimen carácter y funcionalidad a la ciudad donde se programan, ya que el paisaje urbano acaba convirtiéndose en marco permanente para lo escénico, en escenario de representación y en lugar de divertimento. En esta ciudad de los eventos se crean las condiciones para que el público pueda vibrar y sentir una vivencia personal e íntima memorable, al tiempo que vivir una experiencia emocional colectiva asistiendo a acontecimientos que son comercializados globalmente como únicos e irrepetibles.

En la bibliografía abundan las tipologías de eventos, las cuales toman distintos criterios de clasificación (escala territorial, periodicidad, temática, equipamientos, objetivos y costes) (Paül i Agustí, 2013; Richards \& Ruiz, 2017). La más citada es la propuesta por Getz $(2012,2013)$, que los clasifica temáticamente en seis tipos: celebraciones culturales, foros de negocios y comercio, encuentros artísticos y de entretenimiento, competiciones deportivas y recreativas, cumbres políticas y de 
estado y eventos privados. Siguiendo el esquema diseñado por este autor, presentamos una taxonomía que trata de perfilar con mayor precisión este fenómeno para el estudio de caso. Tal como se puede apreciar en la Figura 1, el criterio central por el que hemos optado para establecer la clasificación es el tipo de asistente que participa en los mismos.

FIGURA I | Tipología de acontecimientos mediáticos

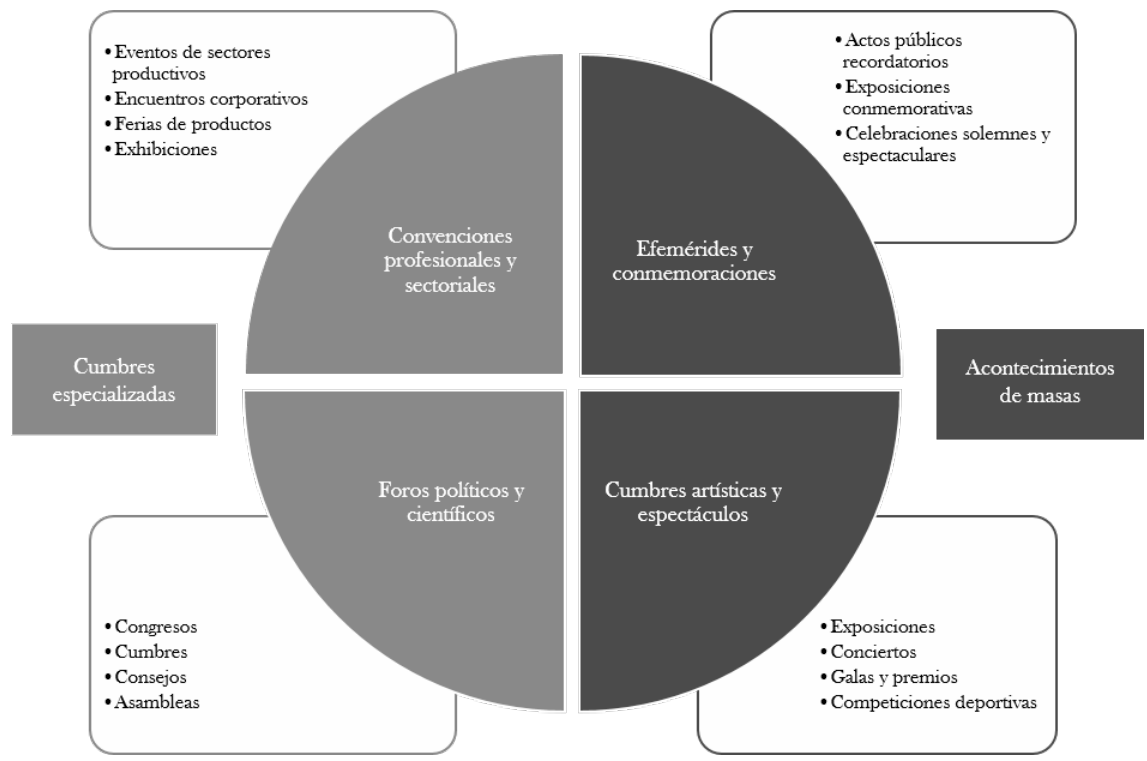

FUENTE: ELABORACIÓN PROPIA

\section{Metodología de la investigación}

Como se ha indicado, este estudio analiza la especialización del gobierno municipal de Sevilla (Andalucía, Espańa) en la organización de un calendario ininterrumpido (casi en cadena) de efímeros eventos de impacto internacional. Esta acción constituye el principal pilar de la estrategia de posicionamiento y crecimiento turístico, al tiempo que una fuente de legitimación social. La investigación pone su foco en el estudio de los discursos emitidos por los responsables públicos y privados en relación con estos acontecimientos, los mismos que han sido difundidos por los medios de comunicación escritos más influyentes de la ciudad a nivel local durante 2018 y 2019. La elección de este periodo se justifica por dos razones fundamentales. En primer lugar porque, tras varias décadas, 2018 y 2019 han sido los ańos en los que se ha materializado un mayor número de grandes acontecimientos internacionales. En segundo, porque esta estrategia constituye desde entonces el eje principal sobre el que pivota la política turística local.

Se han seleccionado los periódicos Diario de Sevilla, ABC de Sevilla y El Correo de Andalucía por su larga trayectoria y consolidación local. De ideología conservadora 
y localista, estos diarios son vendidos en quioscos y pueden hallarse fácilmente en bares, restaurantes, peluquerías y otros establecimientos, donde son leídos libremente por los clientes. ${ }^{2}$ Además, las ediciones electrónicas de los tres rotativos son muy consultadas y es frecuente que las noticias y artículos de opinión más relevantes se reboten en las redes sociales, multiplicando de este modo su repercusión social. Complementariamente, se han estudiado las páginas web de las entidades promotoras, así como noticias aparecidas en algunos de los periódicos internacionales más relevantes (The Guardian, Le Figaro, The Washington Post, Zeit, entre otros).

Como se aprecia en la Tabla 1, la muestra compilada es de 403 noticias en las que autoridades y empresarios destacados del sector valoran alguno de los veintitrés acontecimientos de impacto internacional celebrados en Sevilla durante el periodo 2018-2019.

TABLA I Número de noticias consultadas y analizadas por medios

\begin{tabular}{|l|c|c|}
\hline \multicolumn{1}{|c|}{ MEDIOS } & NÚMERO DE NOTICIAS & PORCENTAJE \\
\hline El Correo de Andalucía & 86 & 21,30 \\
\hline ABC de Sevilla & 141 & 34,90 \\
\hline Diario de Sevilla & 129 & 32,00 \\
\hline Otros & 47 & 11,60 \\
\hline Total & 403 & 100 \\
\hline
\end{tabular}

FUENTE: ELABORACIÓN PROPIA

En la investigación se han utilizado dos técnicas de investigación: el análisis de contenido y la interpretación semiótica. Ambos enfoques son complementarios. El primero ha consistido en registrar y clasificar las noticias, lo que ha exigido la realización de un trabajo previo de recopilación del repertorio existente. Posteriormente se ha procedido a sistematizar la información, entresacando -dentro de su contexto- las declaraciones formuladas por responsables públicos respecto de cada uno de los acontecimientos. Esta selección ha permitido la reducción del abundante corpus documental a unidades significativas y estructuradas.

El análisis semiótico es la segunda técnica utilizada. Consiste en la interpretación de los argumentos presentes en las manifestaciones públicas y en la traducción de la narrativa implícita y explícita que subyace a los discursos, lo que permite profundizar en las ideologías y los objetivos de quienes los formulan. En este trabajo se ha constatado la existencia de una serie de argumentos reiterados en las afirmaciones de los distintos actores, lo que ha permitido clasificarlos y codificarlos de acuerdo con tres tipos de cuestiones básicas: discursos que ponen el acento en la bondad de la estrategia de posicionamiento global; argumentos que ensalzan la especialización en la celebración sucesiva de eventos; y manifestaciones que subrayan los beneficios socioeconómicos derivados de los acontecimientos globales.

2 En septiembre de 2018 desapareció la edición en papel de El Correo de Andalucía, publicándose desde entonces solo en formato digital. 
Es importante resaltar que estos patrones discursivos reiterativos son formulados tanto por responsables empresariales como por políticos del gobierno municipal, así como por la línea editorial de los diarios analizados. Esta coincidencia revela la sintonía ideológica existente en los poderes fácticos locales, los cuales asumen un enfoque turístico-céntrico cuyo guion "hiperneoliberal" hace apología de las presuntas bondades de un sector del que se dice que, indiscutiblemente, intensifica el crecimiento económico y genera empleo como ninguna otra actividad (Amore \& Hall, 2017; Jafari, 2005; Saarinen, 2006).

\section{Los acontecimientos mediáticos globales como piedra angular de la estrategia turística en Sevilla}

Sevilla es una ciudad media, cuya población alcanza los 700.000 habitantes y cuya área metropolitana supera con creces el millón de vecinos. En esta urbe, la actividad turística constituye una de las principales bases económicas. De hecho, la tradición turística viene de antiguo, ya que las primeras guías de viajes y las operaciones urbanísticas dirigidas a crear atmósferas atractivas cargadas de exotismo romántico para los viajeros se remontan a finales del siglo XIX. Esta especialización ha convertido a Sevilla en una de las más destacadas ciudades turísticas del Estado espańol. Lo anterior se constata en la Figura 2, donde se aprecia la tendencia ascendente experimentada en los últimos ańos.

FIGURA 2 | Turistas alojados y pernoctaciones en establecimientos turísticos. Sevilla, 2012-2018

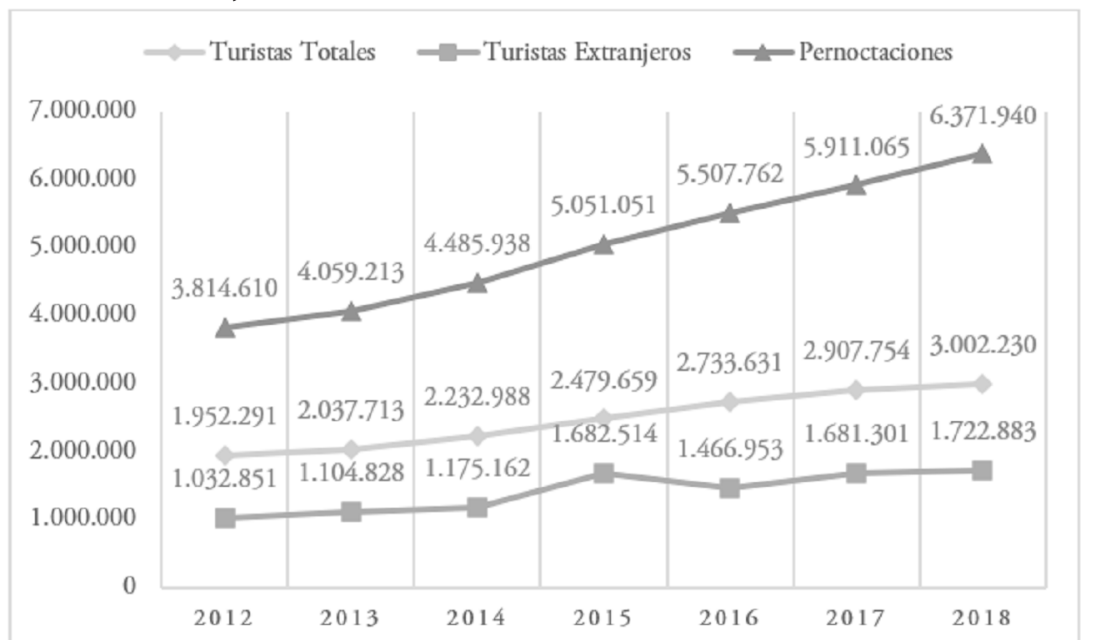

FUENTE: CENTRO DE DATOS TURÍSTICOS, AYUNTAMIENTO DE SEVILLA. ELABORACIÓN PROPIA

Obviamente, la posición de Sevilla en el competitivo marco de ciudades turísticas globales no es tan destacada como la de otras urbes españolas, tales como Barcelona o Madrid. Sin embargo, la apuesta decidida del consistorio en el último quinquenio 
está suponiendo un ascenso en este ranking y un reconocimiento por parte de entidades y consultoras internacionales. De hecho, la ciudad ha sido distinguida con prestigiosos galardones. En 2019, Sevilla fue clasificada como la tercera mejor ciudad europea en el ranking "The Top 15 Cities in Europe", que elabora la prestigiosa revista estadounidense Travel+Leisure (Stubbs, 2019); y en 2018, la editora de guías de viajes Lonely Planet la reconoció con la distinción "Best in Travel" como mejor destino turístico urbano mundial (vV.AA., 2018).

En el equipo gestor del gobierno de Sevilla existe una voluntad política manifiesta de orientar su estrategia hacia la atracción de inversiones y visitantes, para convertir a la ciudad en un destacado lugar de consumo y entretenimiento que estimule a las entidades financieras y empresas multinacionales a dinamizar la actividad local. ${ }^{3}$ Esta dirección política, que en la práctica supone la consolidación del neoliberalismo en el gobierno de la ciudad, es una adaptación local a los procesos de globalización, que permite a las grandes corporaciones penetrar y dominar la actividad turística en los destinos (Brenner \& Theodore, 2005; Fletcher, 2019). De hecho, la propia estructura orgánica municipal se adapta a este objetivo, ya que las competencias atribuidas por el equipo de gobierno al área de turismo local se centran exclusivamente en la "proyección de la imagen de la Ciudad de Sevilla, la promoción exterior de Sevilla y la organización de eventos" (Boletín Oficial de la Provincia [вор], 2019, p. 15). Lo anterior revela que los diversos impactos sociales ocasionados por la actividad no constituyen atribuciones directas del principal órgano turístico municipal. Este enfoque es coherente con el planteamiento neoliberal que parte de una visión benéfica del turismo y reduce el papel del gobierno a favorecer las condiciones para el crecimiento del sector.

Desde el ayuntamiento se asume activamente este rol y no se oculta ante los medios que el turismo es un eje prioritario y estratégico de la acción de gobierno. En este sentido, el propio alcalde declaraba a un medio local:

Entre los objetivos que nos marcamos al inicio del mandato estaba el de mejorar el posicionamiento internacional de la ciudad y el de hacer de Sevilla capital del turismo. (El Correo de Andalucía, 6/11/2017)

En su proyecto de internacionalización, el consistorio ha impulsado todas las iniciativas de singularización mencionadas en la introducción. Es clara su apuesta por la potenciación de una imagen de marca sugestiva a través de las campañas de marketing ("city branding"), así como la labor de estetización de las áreas más antiguas de su casco urbano ("museificación"). Tampoco ha renunciado a proyectar una imagen de modernidad con la erección de grandes edificios emblemáticos e innovadores, que se localizan en zonas de consumo cosmopolita ("neomonumentalismo"). Además, ha organizado importantes megaeventos, entre los que cabe subrayar la Exposición Universal celebrada en 1992, la cual supuso una notable transformación urbanística que ha marcado

3 En el periodo 2015-2019 gobernó el ayuntamiento hispalense una coalición liderada por el Partido Socialista Obrero Español (pSOE). Posteriormente, en las elecciones municipales de 26 de mayo de 2019, el equipo dirigido por el mismo alcalde logró revalidar la alcaldía para el periodo 2019-2022, gobernando esta vez en solitario con un programa de gobierno continuista con respecto al proyecto de grandes fastos iniciado cuatro ańos antes. 
el paisaje urbano. En los últimos años, la iniciativa más relevante es la programación de una agenda de acontecimientos que alcanzan resonancia internacional ("eventización"). Esta última actuación es el objeto de estudio del presente trabajo.

En su firme apuesta por estar presente en el candelero mediático, la administración sevillana se ha embarcado en una estrategia de organización incesante de fastos, que exige de una planificación y una programación muy estudiadas y que tiene como horizonte temporal el corto periodo de una o dos legislaturas. En la Tabla 2 se muestran los acontecimientos internacionales celebrados entre 2018 y 2019, clasificados según la tipología propuesta en la Figura 1. Como se puede apreciar, el número, la relevancia internacional, la diversidad temática y la sucesión de los eventos revelan la especialización de la ciudad en la producción en serie de acontecimientos mediáticos.

TABLA 2 Acontecimientos mediáticos internacionales celebrados en Sevilla (2018-2019)

\begin{tabular}{|c|c|c|c|}
\hline CATEGORÍA & TIPO & EVENTOS & FECHA \\
\hline \multirow{17}{*}{$\begin{array}{c}\text { CUMBRES } \\
\text { ESPECIALI- } \\
\text { ZADAS }\end{array}$} & \multirow{6}{*}{$\begin{array}{l}\text { Encuentros del } \\
\text { sector turístico }\end{array}$} & $\begin{array}{l}\text { Cumbre de la Asociación Europea de Operadores Turísti- } \\
\text { cos (ETOA) }\end{array}$ & $09 / 2018$ \\
\hline & & $\begin{array}{l}\text { Congreso de la Asociación de Agentes de Viajes Británicos } \\
\text { (ABTA) }\end{array}$ & $10 / 2018$ \\
\hline & & $\begin{array}{l}\text { Cumbre Europea-China de la Federación Mundial de } \\
\text { Ciudades Turísticas (wTCF) }\end{array}$ & $12 / 2018$ \\
\hline & & $\begin{array}{l}\text { Cumbre Mundial del Turismo del Consejo Mundial de } \\
\text { Viajes y Turismo (wTCF) }\end{array}$ & $04 / 2019$ \\
\hline & & v Cumbre Mundial de Asociaciones de Agencias de Viajes & $11 / 2019$ \\
\hline & & Feria Internacional de Turismo Premium & $11 / 2019$ \\
\hline & \multirow{7}{*}{$\begin{array}{l}\text { Convenciones } \\
\text { profesionales y } \\
\text { de sectores } \\
\text { productivos }\end{array}$} & $\begin{array}{l}\text { Cumbre Europea de la Asociación de Profesionales de la } \\
\text { Gestión de Convenciones (РСМA) }\end{array}$ & $09 / 2018$ \\
\hline & & Cumbre Internacional de Aerolíneas de Bajo Coste (CAPA) & $10 / 2018$ \\
\hline & & Salón Internacional del Caballo, 2018 (sICAB) & $11 / 2018$ \\
\hline & & III Salón Internacional de la Minería (ммн) & $10 / 2019$ \\
\hline & & Foro Industrial Aeroespacial. Space Forum & $10 / 2019$ \\
\hline & & Salón Internacional del Caballo, 2019 (sICAB) & $11 / 2019$ \\
\hline & & Cumbre de la Red de Líderes de Operaciones Aéreas (CAPA) & $12 / 2019$ \\
\hline & \multirow{4}{*}{$\begin{array}{l}\text { Foros políticos y } \\
\text { científicos }\end{array}$} & $\begin{array}{l}\text { v Congreso Mundial de Estudios de Oriente Medio y el } \\
\text { Norte de África (wocmes) }\end{array}$ & $07 / 2018$ \\
\hline & & II Foro Global de Gobiernos Locales & $01 / 2019$ \\
\hline & & Asamblea Regional y Local Euro-mediterránea (ARLEM) & $02 / 2019$ \\
\hline & & Consejo Ministerial de la Agencia Espacial Europea (ESA) & $11 / 2019$ \\
\hline \multirow{6}{*}{$\begin{array}{c}\text { ACONTECI- } \\
\text { MIENTOS } \\
\text { MASIVOS }\end{array}$} & \multirow{2}{*}{$\begin{array}{l}\text { Efemérides y } \\
\text { conmemoraciones }\end{array}$} & $\begin{array}{l}\text { Año Murillo. Iv Centenario del nacimiento del pintor } \\
\text { barroco }\end{array}$ & 2018 \\
\hline & & v Centenario de la Primera Circunnavegación a la Tierra & 2019 \\
\hline & \multirow{4}{*}{$\begin{array}{l}\text { Cumbres artísticas } \\
\text { y espectáculos }\end{array}$} & xx Bienal de Flamenco & $09 / 2018$ \\
\hline & & xv Festival de Cine Europeo de Sevilla & $11 / 2018$ \\
\hline & & $\begin{array}{l}\text { XXvi Gala de los Premios Europeos de la Música (EMA) de } \\
\text { la cadena MTV }\end{array}$ & $11 / 2019$ \\
\hline & & xvi Festival de Cine Europeo de Sevilla & $11 / 2019$ \\
\hline SE & $\begin{array}{l}\text { LUYEN LOS CO } \\
\text { OS DE ELLOS DF } \\
\text { ES TRADICIONA }\end{array}$ & $\begin{array}{l}\text { GRESOS, FERIAS Y ESPECTÁCULOS DE ÁMBITO ANDALUZ } \\
\text { GRAN REPERCUSIÓN MEDIÁTICA. TAMPOCO SE INCLUYEN LAS } \\
\text { ES, QUE ATRAEN MILLARES DE TURISTAS CADA AÑO. }\end{array}$ & $\begin{array}{l}\text { Y ESTATAL, } \\
S \text { FIESTAS Y }\end{array}$ \\
\hline
\end{tabular}


En las siguientes páginas se analizan los discursos emitidos por responsables públicos en torno a los acontecimientos mediáticos internacionales celebrados en la ciudad entre 2018 y 2019. Se han seleccionado aquellas citas que mejor resumen la voluntad de promover y apoyar estos eventos. Esta antología obedece a un esfuerzo de generalización y síntesis, por lo que se han resaltado aquellas declaraciones más representativas, reiteradas y expresivas. Por consiguiente, las citas que se presentan constituyen referentes empíricos paradigmáticos que pretenden compendiar las declaraciones más sustanciales y significativas.

\section{Resultados. Análisis de los discursos}

\section{Estar en el epicentro}

Situar la ciudad en la agenda internacional como un lugar conocido y reconocido es uno de los argumentos habituales que se esgrimen ante la opinión pública como indiscutible justificación de los grandes acontecimientos mediáticos. En estos discursos se repite con expresiones sencillas, pero de fuerte impacto, que el evento posiciona ventajosamente a la ciudad. Por ejemplo, Juan Espadas, alcalde de Sevilla, declaró que la celebración de la Gala de los premios de la cadena de televisión estadounidense MTV:

(...) nos sitúa en el mapa a nivel internacional como un lugar de celebración de eventos de gran calado que significa, si me lo permiten, empezar a jugar en Champion. (Cadena Ser, 26/03/2019)

Este mismo argumento ha sido utilizado en otras ocasiones. Por ejemplo, un mes después de la anterior manifestación se celebró en la ciudad la Cumbre Mundial de Turismo de la World Travel \& Tourism Council (WTTC). El evento, que es considerado el principal encuentro internacional de las grandes corporaciones del sector y calificado en los medios como "el Davos del turismo" (Diario de Sevilla, 1/04/2019), fue anunciado triunfalmente por el mismo alcalde con las siguientes palabras:

La capital juega desde hoy en otra liga, en otra división, en la que hasta ahora solo han estado Madrid y Barcelona. (Diario de Sevilla, 3/04/2019)

Este tipo de declaraciones no son exclusivas de los líderes políticos, sino que las emiten también representantes del empresariado, lo que revela la sintonía y la colaboración de la patronal con la política turística local. Sirva como ilustración la declaración del presidente de la Asociación Empresarial de Agencias de Viajes (AEVISE) con respecto a la celebración sucesiva de congresos internacionales de este ramo en la ciudad:

Estos eventos determinan la inmejorable situación de Sevilla y la colocan en el epicentro del turismo mundial. (Diario de Sevilla, 20/04/2018)

La expresión "estar en el epicentro" utilizada en la anterior declaración es una fórmula retórica muy repetida. En un sentido metafórico, el evento se asemeja a un temblor sísmico cuya sacudida convierte a la ciudad en centro de atención internacional. Esta frase hecha es también de uso común por los responsables políticos para 
subrayar que la intensa magnitud "sísmica" (mediática) derivada de las celebraciones otorga centralidad a la ciudad en el sector turístico. Así, con motivo de la Gala de los Premios Europeos de la Música de la cadena MTv, el delegado municipal de Cultura y Turismo resaltaba los efectos positivos derivados de este evento, pues según su opinión situaba a la ciudad en el punto de mira de la atención internacional gracias a una eficaz política orientada a la celebración periódica de festivales musicales de repercusión global:

Es una promoción impagable, habría que preguntarse cuánto podría costar a las arcas públicas hacer una campaña similar de difusión de la ciudad como destino ideal para cualquier tipo de actividad que convierte su patrimonio en protagonista (...). Sevilla va a convertirse en epicentro del mundo de la música para acoger a productores, programadores y profesionales del sector de primer orden que vienen a conocer nuestra capacidad para organizar eventos. Y lo hacemos (...) continuando un calendario de actividades de diversa naturaleza que probablemente resulte único. $(A B C, 3 / 11 / 2019)$

En las declaraciones se suele apelar también a los sentimientos, al orgullo local y al entronque de dichos acontecimientos con la identidad de la ciudad y la continuidad de su espíritu histórico. En este sentido son significativas las siguientes manifestaciones del máximo responsable municipal con motivo de la celebración de la Cumbre Mundial del Turismo:

Las ciudades con vocación universal como esta tienen que tener ambición para estar en el centro del mapa del mundo. (Diario de Sevilla, 3/04/2019)

Con un estilo épico, en otras ocasiones se subraya que la celebración de estas ceremonias cosmopolitas permitirá recuperar la grandeza, dignidad y centralidad internacional que Sevilla alcanzó en el siglo xvi. En este sentido, son ilustrativas las declaraciones del delegado municipal de turismo en relación con la celebración de la Cumbre Mundial del Turismo:

Queremos volver al epicentro del mapa del mundo y la wTTC nos brinda una oportunidad de oro. (Diario de Sevilla, 1/04/2019)

Dos años antes el alcalde utilizó el mismo argumento para anunciar la celebración del I Foro Global de Gobiernos Locales, ensalzando el papel de la capital hispalense "como ciudad milenaria, puerta de América con una vocación universal que se retoma con esta cita” (El Correo de Andalucía, 23/11/2017).

Otras veces las manifestaciones revelan con claridad meridiana que, con estas actuaciones, la política local persigue posicionar ventajosamente a Sevilla en la red de ciudades globales. Por ejemplo, al hilo de la celebración de la Cumbre Mundial del Turismo, el máximo responsable municipal del sector afirmó con orgullo:

No todas las ciudades pueden incorporar a su currículum la celebración de este tipo de eventos. Los retos del turismo del siglo xxi no se van a analizar ni en Nueva York ni en París, sino en Sevilla, que suma y sigue. (Diario de Sevilla, 1/04/2019) 
Con frecuencia, en las declaraciones se alardea públicamente de que determinados eventos no solo situarán favorablemente a la ciudad en el ranking urbano global, sino que la convertirán en la capital mundial de un determinado sector o actividad cultural. Así, son habituales las afirmaciones optimistas y grandilocuentes que subrayan el ascenso de la ciudad gracias a la celebración de eventos que permitirán "convertir a Sevilla en la capital del turismo" (declaraciones del regidor, $A B C$, 06/11/2017). Pero manifestaciones de este tipo no solo provienen de los responsables públicos y empresariales, sino también de los propios medios de comunicación. Por ejemplo, con motivo de la Cumbre Mundial de la Travel Agencies Associations, el Diario de Sevilla encabezaba la edición con el siguiente titular: "Sevilla se convierte en la capital mundial de las agencias de viajes" (15/10/2019). Este tipo de noticia, que ilustra el apoyo explícito a la estrategia municipal, no es infrecuente en los medios locales, lo que sin duda favorece la creación de un clima social propicio a esta política orientada a la captación de inversores y flujos turísticos.

\section{Sevilla, suma y sigue}

La administración local negocia directamente con los interlocutores internacionales (grandes cadenas televisivas, empresas multinacionales y entidades públicas y privadas supranacionales) ser la sede de futuras ediciones de grandes acontecimientos, para lo que ofrece sus instalaciones y todo tipo de facilidades. Esta orientación exige la formación dentro del consistorio de un cuerpo de profesionales especializados en la gestión de eventos que operan directamente en la captación de acontecimientos internacionales, ofertando la candidatura de la ciudad en aquellos encuentros donde se decide la localización de la sede del espectáculo (Figura 3). Así lo explicaba el concejal de Turismo a un medio local:

Un gran congreso (...) requiere de una tarea previa y el estar presente donde se van a decidir las futuras ciudades donde se van a albergar ese tipo de congresos. Eso requiere un trabajo laborioso, callado, si ustedes quieren, de día a día, de influencia, de presentar candidatura, de hacer el seguimiento... y os puedo decir que en este momento los equipos de marketing del Consorcio de Turismo de Sevilla (...) están trabajando para estar presentes en esos circuitos donde se deciden los grandes eventos para los próximos años. (El Correo de Andalucía, TV)

Posteriormente, el mismo responsable político se jactaba ante los medios de haber negociado en la World Travel Market de Londres que Sevilla fuera seleccionada como la futura sede de la Cumbre Internacional del Turismo Asiático (European Summit, Sevilla 2020), organizada por la Pacific Asian Travel Association (PATA), la gran asociación del turismo de Asia:

Ya ha quedado cerrado y vendrán representantes de agencias, hoteles, turoperadores y empresas de toda el área Asia Pacífico, lo que va a suponer un verdadero punto de inflexión para la ciudad en lo que tiene que ver con estos mercados de larga distancia tan emergentes y de los que tanto provecho podemos sacar con este buen momento que atraviesa la ciudad. $(A B C, 7 / 11 / 2019)$ 
FIGURA 3 | Acto de presentación de la candidatura sevillana a la cumbre global de la wTTC (Buenos Aires, Argentina, 2018)

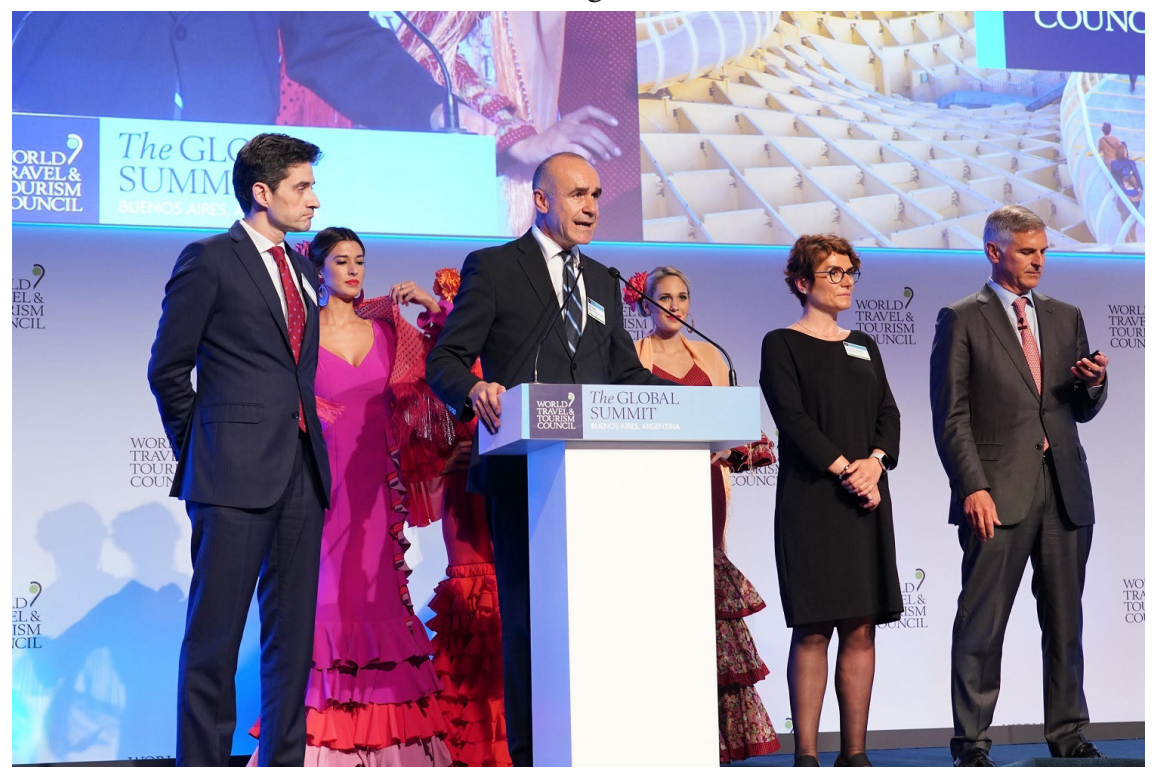

FUENTE: WORLD TRAVEL TOURISM COUNCIL (WTTC) (2OI8)

El convencimiento de la repercusión positiva que tienen los eventos explica el esfuerzo de los gestores municipales por asegurar una agenda ininterrumpida de grandes citas. De ahí que se considere un éxito establecer acuerdos de celebración de varias ediciones sucesivas del mismo evento. Cuando esto se consigue, la noticia es transmitida a los medios como un auténtico triunfo. Por ejemplo, tras el contrato suscrito con la entidad Emotions Travel Community para celebrar durante tres ańos sucesivos la Feria Internacional del Turismo Premium en Sevilla (2019, 2020 y 2021), el alcalde declaró orgulloso ante los medios:

Meternos en este mundo es un salto cualitativo, porque creo que lo hemos demostrado todo en el turismo de congresos y de eventos en esta ciudad. Sevilla hoy es una de las ciudades más competitivas, por no decir la que más, a la hora de captar citas de alcance de todos los ámbitos. (ABC, 12/11/2019)

Las autoridades explican ante los medios que el objetivo de cada nueva actuación es reforzar el posicionamiento de la ciudad y consolidarla como un escenario de conmemoraciones, espectáculos y congresos a nivel internacional. En este sentido, con motivo de la celebración de la vigesimosexta edición de la Gala de los premios Europe Music Awards (EMA) de la cadena estadounidense MTV, el alcalde declaró con optimismo:

Este nuevo evento forma parte de la estrategia de posicionamiento de la ciudad, que no solo es un gran referente turístico, sino también de celebración de grandes acontecimientos. (ABC, 26/03/2019) 
Otra metáfora utilizada con insistencia es la de "recurso". Esta argumentación se ilustra con sencillas asociaciones que, en sentido figurado, comparan la actividad extractiva con la celebración de eventos como vía para el crecimiento y la captación de nuevos flujos turísticos. Por ejemplo, con estas palabras el responsable de turismo del consistorio explicaba la estrategia de producción de eventos en serie:

Es el filón que el Ayuntamiento de Sevilla quiere explotar y repetir (...). Hace ya dos años la ciudad logró ser sede de un encuentro similar y el esfuerzo se ha traducido en un notable incremento en las cifras de visitantes. (ABC, 8/10/2018)

\section{Vender Sevilla}

Los responsables públicos, los medios de comunicación mayoritarios, el sector empresarial, así como gran parte de la opinión pública consideran que el turismo impulsa e intensifica el crecimiento económico, genera empleo como ningún otro sector y desencadena una espiral de actividades que diversifica la economía y la dinamiza gracias a la atracción de capitales privados externos. Este efecto multiplicador o de arrastre sobre el resto de la economía es el que se arguye como justificación de toda iniciativa orientada a la captación de flujos turísticos y financieros. De ahí que el turismo se haya convertido en uno de los pilares centrales de la política local, tal como reconocía el mismo alcalde ante los medios:

Hemos concebido el turismo en la ciudad como un canal de captación de inversiones para el propio ámbito turístico, pero también para el conjunto de la economía. $(A B C, 07 / 11 / 2019)$

Esta visión optimista es la que se esgrime también para justificar la estrategia de celebración de eventos internacionales. En coherencia con lo anterior, las declaraciones de los responsables públicos y empresariales, así como los editoriales y artículos de opinión contenidos en los medios de comunicación más importantes de la ciudad, coinciden en el carácter benéfico que supone la celebración de eventos. Todos concuerdan en que esta apuesta es una estrategia indiscutiblemente positiva en términos económicos, laborales y sociales.

No obstante, es interesante resaltar que las referencias cuantitativas respecto a las cifras de creación de empleo, los ingresos del sector turístico o la dinamización y diversificación económica sean escasas, poco precisas, ambiguas y, a veces, contradictorias. Así, por ejemplo, es habitual que los titulares de los rotativos subrayen -con tipografía de gran tamaño y en páginas centrales- los beneficios derivados de la celebración de los eventos, tomando como única fuente de información las afirmaciones formuladas por los organizadores. Por ejemplo, con motivo de la celebración de la Cumbre Mundial del Turismo de la WTTC, el titular de un diario local destacaba que era:

Una oportunidad de negocio de 1.700 millones de euros a la vista. La cumbre mundial dejará en Sevilla ingresos directos de al menos 3,5 millones, sin contar con el gran marketing exterior. (Diario de Sevilla, 1/04/2019) 
Con este estilo entusiasta, los medios transmiten literalmente las declaraciones de los responsables políticos y empresariales, pero sin contrastar la información ni realizar un posterior seguimiento y evaluación de la repercusión socioeconómica objetiva del acontecimiento. Del mismo modo, la generación de empleo es otro de los argumentos más trillados para justificar la organización de eventos, ya que se asegura que es una medida adecuada para contrarrestar la alta tasa de desempleo registrada en la ciudad ( $21 \%$ en octubre de 2019$)$. Sin embargo, tampoco se aportan datos fidedignos que permitan comprobar la veracidad de dichas afirmaciones y determinen el volumen objetivo de empleo generado.

La única referencia contrastable que aporta la hemeroteca es la referida al incremento sostenido del número de turistas (ver Figura 2), pues las fuentes en las que se apoya son las estadísticas oficiales. Pero lo que no está verificado es la relación entre acontecimientos y crecimiento de las llegadas. Sin embargo, con énfasis casi triunfal, los líderes empresariales y políticos establecen una correlación automática entre aumento del número de visitantes y celebración de eventos internacionales, sin aportar elementos que permitan constatar dicha correspondencia. Una declaración que ilustra bien este razonamiento fue la emitida por el máximo responsable local de turismo, en las que vinculaba directamente el incremento de turistas en octubre de 2019 con los eventos celebrados:

Se trata del mejor mes de la serie estadística del turismo en nuestra ciudad y estuvo impulsado por la agenda musical previa a la gala de entrega de los premios europeos MTV y el conjunto de la oferta cultural, los eventos deportivos como el triatlón o las carreras populares y las citas como el Salón del Motor de Ocasión o los congresos internacionales de minería y salud pulmonar, sin olvidar la nueva temporada otoño-invierno del aeropuerto de San Pablo con más conexiones aéreas que nunca.

(Diario de Sevilla, 3/12/2019)

Un argumento repetido es que todo evento internacional supone no solo un incremento del volumen de turistas, sino además un indiscutible impulso al desarrollo de la ciudad. Aquí también se suele recurrir a metáforas efectistas y sensacionalistas, como la que asocia cada evento a un "trampolín", el cual presta impulso a la ciudad para dar el gran salto que permita diversificar su tejido empresarial. Una muestra de ello fueron las declaraciones del alcalde anunciando la Cumbre Mundial del Turismo de la wTTC:

Será un trampolín para generar iniciativas productivas en Sevilla, una ciudad atractiva y con futuro para ser visitada y, ¿por qué no?, para ser sede de empresas. ( $E l$ Pais, 22/01/2019)

La anterior declaración es significativa. Revela que para los responsables públicos el principal objetivo de la celebración de eventos es atraer capitales y captar inversores que instalen empresas y/o adquieran bienes inmobiliarios. En esta estrategia, los eventos son contemplados principalmente como oportunidades de negocio, pues permiten situar a la ciudad en el escaparate mediático internacional como un escenario sugestivo para la inversión extranjera. Esto es especialmente evidente en las grandes cumbres sectoriales internacionales, donde empresarios de distintas ramas 
(aerolíneas, agencias de viajes, cadenas hoteleras, fondos de inversión, minería, industria, etcétera) establecen una intensa agenda de encuentros bilaterales. El contexto es aprovechado por los responsables públicos para comercializar la ciudad, mostrando las ventajas que supone invertir en la misma e informar de la oferta existente, así como de las facilidades que se les ofrecen. Esta intención es reconocida abiertamente y sin tapujos en los medios de comunicación. Sirvan como ejemplo las declaraciones de la delegada municipal de Relaciones Institucionales, Economía y Comercio:

Buscamos atraer inversiones a Sevilla, así como apoyar y proyectar internacionalmente su tejido empresarial y captar fondos de proyectos europeos. (El Correo de Andalucía, 18/09/2019)

La acción pública de esta promoción no es improvisada, sino que obedece a una estrategia claramente definida y estudiada. Con lemas tales como "Una ciudad para invertir, crear, innovar, visitar y vivir" (Diario de Sevilla, 23/03/2019), los gestores de eventos informan a los potenciales inversores internacionales que participan en los encuentros y congresos de la disponibilidad de suelo urbano, anuncian las ventajas derivadas de invertir en sectores emergentes y pujantes de la ciudad (turismo, energías renovables, salud, TIC, economía digital...) y muestran la oferta de inmuebles singulares de titularidad municipal ( $A B C, 3 / 04 / 2019)$.

Esta estrategia hace visible la participación de los responsables públicos en la ideología neoliberal, que entiende que la atracción de capitales exteriores para la adquisición de suelo, la generación de negocios y el impulso de actividades especulativas es la vía más acertada para promover el crecimiento económico. Dentro de este marco ideológico, la organización de cumbres y espectáculos funciona prioritariamente como un reclamo para captar inversores, lo que incluso puede anteponerse al sentido (cultural, profesional, político o conmemorativo) con el que se conciben estos eventos globales. En palabras del delegado municipal de Hábitat Urbano, Cultura y Turismo:

Nunca antes la ciudad ha estado tanto en el foco internacional y no solo desde el punto de vista turístico, sino también empresarial y de captación de inversiones, dado que las cumbres internacionales tienen también sus lógicas repercusiones en la inversión extranjera y, por tanto, sobre el conjunto de la economía local y su empleo. (Diario de Sevilla, 8/04/2018)

Sin embargo, la oferta de suelo e inmuebles es una estrategia que potencialmente entraña sus riesgos, pues puede acelerar el proceso de globalización del mercado inmobiliario local y detraer recursos públicos, favoreciendo la práctica de acumulación por desposesión (Harvey, 2004), lo que, además, entraña serias amenazas de alimentar procesos especulativos que relancen la burbuja inmobiliaria (Delgado, 2019).

\section{Conclusiones. ¿Fastos nefastos?}

En este trabajo se ha analizado un caso concreto de eventización en el marco de los procesos de festivalización urbana que se expanden a escala planetaria. Con el estudio se ha tratado de contribuir al debate y al análisis crítico de la eventización 
en contextos urbanos. La investigación ha revelado cómo la política municipal desarrollada en Sevilla se ha especializado en la producción en cadena de espectáculos y cumbres internacionales.

Esta política constituye en la actualidad el eje principal de la estrategia de posicionamiento en el archipiélago de ciudades globales y representa un cambio de dirección de la actuación municipal con respecto a etapas pasadas. Supone el tránsito de una política de base local a otra que proyecta al lugar en una economía de flujos muy interconectada, jerarquizada e inestable, la cual es característica de la actual era de la hipermovilidad (Elliot \& Urry, 2010; Hannerz, 1998; Sheller, 2017; Sheller \& Urry, 2006). En esta evolución, la tradicional función de la administración local como institución prestadora de servicios, que dota a la sociedad de infraestructuras y equipamientos públicos, se complementa o es reemplazada por otro sistema en el que la ciudad es gestionada como una empresa y el gobierno municipal desempeña el papel de promotor especializado en la captación de inversiones del exterior y de interlocutor para la materialización de negocios privados (González \& Mantecón, 2014; Harvey, 1989).

Esta reorientación de las políticas municipales desde lo local a lo global se produce en el marco de la estrategia neoliberal que promueve la penetración del capital externo en la economía local. En Sevilla, el gobierno municipal apuesta decididamente por apoyar y promover todas las iniciativas que faciliten el desarrollo de estas dinámicas, cumpliendo punto por punto lo esperado por las lógicas globales, con los acontecimientos mediáticos como la principal iniciativa que se desarrolla en este sentido en los últimos ańos. Sin embargo, la amenaza más importante que puede suponer este enfoque empresarial de la administración municipal es que los objetivos de captación de capitales y visitantes se antepongan a la satisfacción de las necesidades básicas de la población local, lo que implica priorizar la inversión pública hacia una política que desvía el presupuesto de lo social y local en beneficio del espectáculo y lo global.

Del análisis de los discursos emitidos es importante resaltar la enorme coincidencia de los distintos responsables locales (políticos, empresarios y medios de comunicación) en considerar los eventos mediáticos como instrumentos idóneos para el crecimiento. Es más, se ha comprobado que, cada vez que se anuncia o evalúa la celebración de un acontecimiento, los argumentos esgrimidos por estos actores son repetidos, utilizando a menudo los mismos términos y figuras retóricas, lo que revela el alto grado de consenso existente. Obviamente, el relevante apoyo del sector turístico, de los propios medios de comunicación, así como el amplio respaldo de los grupos políticos de la oposición en esta materia favorecen la celebración de eventos y la aplicación de la agenda política neoliberal. Por otro lado, el análisis de las declaraciones revela además que esta "cultura del evento" tiene una orientación de consumo interno no menos importante, ya que la buena imagen cosechada por los grandes acontecimientos funciona como una eficaz arma propagandística con fines electorales, pues publicita una presunta buena acción de gobierno, al tiempo que vela dejaciones, carencias e inacciones.

Más allá del caso de estudio aquí analizado, el debate político, académico y ciudadano de fondo que subyace es si la eventización es un medio para impulsar el 
desarrollo, revitalizar y transformar la ciudad en un lugar creativo, atractivo y habitable, por cuanto fomenta la participación ciudadana, contribuye a generar orgullo y fortalece el sentido de comunidad entre los residentes, tal como proponen numerosos autores (Escudero, 2019; Ganau, 2007; Herrero et al., 2006; Paül i Agustí, 2013; Richards \& Palmer, 2010; Richards \& Ruiz, 2017; Richards \& Wilson, 2004; Sharpley \& Stone, 2012); o si, por el contrario, es un instrumento para la expansión y acumulación del capital (Gotham, 2005; Harvey, 1988; Mascarenhas, 2007) que aleja la atención política de los problemas sociales cotidianos (Evans, 2003; Shin, 2012), transforma las actividades y espacios de ocio en espectáculos corporativos y privatizados (Hannam, 2018; Spracklen et al., 2013) y propicia la gentrificación de las áreas donde se celebran (Fessler \& Berenstein, 2006). En definitiva, un reto para investigaciones futuras consiste en verificar si los eventos sirven al lugar o si el lugar sirve a los eventos tan solo como un simple telón de fondo (Brito \& Richards, 2017; Van Alalst \& van Melik, 2012). Y esto es un problema empírico.

No hay duda de que en una sociedad en red es imprescindible que las ciudades estén interconectadas y, como se ha mostrado, los eventos internacionales favorecen estos procesos. Sin embargo, de la acción política y ciudadana depende que estas actuaciones de internacionalización conecten también con la sociedad local, produciendo una amplia gama de externalidades (Brito \& Richards, 2017) que reviertan los beneficios de los acontecimientos mediáticos en la calidad de vida de los residentes y en el desarrollo de una economía de la cultura.

\section{Agradecimientos}

Este estudio ha sido realizado en el marco del Proyecto "Desarrollo urbano e impactos socio-espaciales del sector turístico en grandes ciudades andaluzas". Proyecto I+D+I del PAIDI-FEDER 2020; código P I 8-RT-2427.

\section{Referencias bibliográficas}

Amore, A. \& Hall, C. M. (2017). National and urban public policy in tourism. Towards the emergence of a hyperneoliberal script? International Journal of Tourism Policy, 7(1), 4-22. https://doi.org/10.1504/IJTP.2017.082761

Boletin Oficial de la Provincia (вор). (2019). Sevilla: Organización del gobierno municipal. Boletin Oficial de la Provincia, 165, 12-18. https://www.dipusevilla.es/system/modules/ com.saga.sagasuite.theme.diputacion.sevilla.corporativo/handlers/download-bop. pdf?id=407a4696-a886-11e9-ae74-0050569fe27b

Brenner, N. \& Theodore, N. (2005). Neoliberalism and the urban condition. City, 9(1), 101107. https://doi.org/10.1080/13604810500092106

Brito, M. \& Richards, G. (2017). Guest editorial. International Journal of Event and Festival Management, 8(1), 2-7. https://doi.org/10.1108/IJEFM-01-2017-0007

Cudny, W. (2016). Festivalisation of urban spaces. Factors, processes and effects. Springer.

Debord, G. (1992). La société du spectacle. Gallimard. 
Delgado, M. (2019). Las cuentas del turismo corporativo en Andalucía. Portal de Andalucía. https://portaldeandalucia.org/opinion/las-cuentas-del-turismo-corporativo-enandalucia/

Elliot, A. \& Urry, J. (2010). Mobile lives. Routledge.

Escudero, L. A. (2019). Los eventos distintivos en grandes destinos del turismo cultural. El caso del Año Greco en Toledo (España). Cuadernos de Turismo, (44), 129-164. https://doi. org/10.6018/turismo.44.404791

Evans, G. (2003). Hard-branding the cultural city - from Pardo to Prada. International Journal of Urban and Regional Research, 27(2), 417-440. https://doi.org/10.1111/14682427.00455

Fessler, L. \& Berenstein, P. (2006). Contemporary urban spectacularisation. En J. Monclús \& M. Guardia (Eds.), Culture, urbanism and planning (pp. 241-254). Ashgate.

Fletcher, R. (2019). Neoliberalismo y turismo. En E. Cañada \& I. Murray (Eds.), Turistificación global. Perspectivas criticas en turismo (pp. 37-52). Icaria.

Gago, C., Córdoba, J. \& Díez, R. (2017). Los listados de ciudades globales. Desde la práctica investigadora a su utilización como argumento en la planificación urbana neoliberal. Revista Internacional de Sociología, 75(1, e054). https://doi.org/10.3989/ ris.2017.75.1.15.11

Ganau, J. (2007). El papel de la cultura en el cambio económico y la promoción de las ciudades. El caso de Philadelphia. Scripta Nova, 11, 245(61). https://www.raco.cat/index.php/ ScriptaNova/article/view/74087

Getz, D. (2012). Event studies. Theory, research and policy for planned events. Routledge.

Getz, D. (2013). Event tourism. Concepts, international case studies, and research. Cognizant Communication Corporation.

González, S. (2011). Bilbao y Barcelona 'in Motion'. How urban regeneration 'models' travel and mutate in the global flows of policy tourism. Urban Studies, 48(7), 1397-1418. https://doi.org/10.1177/0042098010374510

González F. \& Morales, S. (2009). Ciudades efimeras. Transformando el turismo urbano a través de la producción de eventos. Universitat Oberta de Catalunya (UOc).

González, F. \& Morales, S. (2017). El impacto cultural y social de los eventos celebrados en destinos turísticos. La percepción desde el punto de vista de los organizadores. Cuadernos de Turismo, (40), 339-362. https://doi.org/10.6018/turismo.40.309741

González, R. \& Mantecón, A. (2014). Turismo y negocio inmobiliario: la crisis de un modelo de desarrollo. Tres estudios de casos de Canadá, Argentina y Espańa. Estudios y Perspectivas en Turismo, 23(4), 685-705. https://rua.ua.es/dspace/bitstream/10045/40964/1/2014_ Gonzalez_Mantecon_EPT.pdf

Gotham, K. (2005). Theorizing urban spectacles: Festivals, tourism and the transformation of urban space. City, 9(2), 225-246. https://doi.org/10.1080/13604810500197020

Gravari-Barbas, M. (2007). À la conquête du temps urbain: la ville festive des 24 heures sur 24. En P. Duhammel \& R. Knafou (Eds.), Mondes urbains du tourisme (pp. 55-74). Belin.

Hall, C. M. (2006). Urban entrepreneurship, corporate interest and sports mega-events: the thin policies of competitiveness within the hard outcomes of neoliberalism. The Sociological Review, 54(s2), 59-70. https://doi.org/10.1111/j.1467-954X.2006.00653.x

Hannam, K. (2018). Mobilities and eventization: Dubai as a city of speed. Via Tourism Review, (14). https://doi.org/10.4000/viatourism.3367 
Hannerz, U. (1998). Conexiones transnacionales. Cultura, gente, lugares. Frónesis.

Harvey, D. (1988). Voodoo cities. New Statesman and Society, (30), 33-35.

Harvey, D. (1989). From managerialism to entrepreneurialism: the transformation in urban governance in late capitalism. Geografiska Annaler. Series B, Human Geography, 71(1), 3-17. https://doi.org/10.1080/04353684.1989.11879583

Harvey, D. (2004). El 'nuevo' imperialismo. Acumulación por desposesión. Socialist Register, (40), 63-87. https://socialistregister.com/index.php/srv/article/view/14997

Herrero, L. C., Sanz, J. Á., Devesa, M., Bedate, A. \& Del Barrio, M. J. (2006). The economic impact of cultural events: A case study of Salamanca 2002, European capital of culture. European Urban and Regional Studies, 13(1), 41-57. https://doi. org/10.1177/0969776406058946

Jafari, J. (2005). El turismo como disciplina científica. Politica y Sociedad 42(1), 39-56. https:// revistas.ucm.es/index.php/POSO/article/view/POSO0505130039A

Jago, J. \& Shaw, R. (1998). Special events: A conceptual and definitional framework. Festival Management and Event Tourism, 5(1), 21-32. https://doi.org/10.3727 /106527098792186775

Lamond, I. \& Platt, L. (Eds.). (2016). Critical event studies: approaches to research. Palgrave Macmillan.

Lipovetski, G. \& Seroy, J. (2015). La estetización del mundo. Vivir en la época del capitalismo artístico. Anagrama.

Mascarenhas, G. (2007). Mega-eventos esportivos, desenvolvimento e cidadania: Uma análise da gestâo da cidade do Rio de janeiro por ocasiâo dos jogos Pan-Americanos-2007. Scripta Nova, 11, 245(13). https://www.raco.cat/index.php/ScriptaNova/article/ view/74040

Muñoz, F. (2008). Urbanalización. Paisajes comunes, lugares globales. Gustavo Gili.

Négrier, E. (2011). La festivalización de la cultura: una dialéctica de los cambios de paradigma. En Ll. Bonet \& H. Schargorodsky (Dirs.), La gestión de festivales escénicos: conceptos, miradas y debates (pp. 17-30). Cuadernos Gescénic-6.

Paül i Agustí, D. (2013). Las políticas culturales y sus repercusiones en la imagen de la ciudad. Scripta Nova, 17(432). http://www.ub.edu/geocrit/sn/sn-432.htm

Pisonero, R. D. (2017). Los megaeventos: indicadores del archipiélago mundial desde una perspectiva cultural basada en el consumo de eventos de ocio. Revista EURE - Revista de Estudios Urbano Regionales, 43(128), 197-228. http://dx.doi.org/10.4067/S025071612017000100009

Richards, G. \& Colombo, A. (2017). Creating network value: Sónar Festival Barcelona as a global events hub. En J. Armbrecht, E. Lundberg, T. Anderssony \& D. Getz (Eds.), The value of events (pp. 73-86). Routledge.

Richards, G. \& Palmer, R. (2010). Eventful cities: Cultural management and urban revitalisation. Butterworth-Heinemann.

Richards, G. \& Ruiz, A. (Coords.). (2017). Experiencias turisticas de festivales y eventos. PASOS, Revista de Turismo y Patrimonio Cultural (RTPC). Colección PASOS edita no 17. https://issuu.com/pasosonline/docs/psedita_17

Richards, G. \& Wilson, J. (2004). The impact of cultural events on city imagen: Rotterdam, cultural capital of Europe 2001. Urban Studies, 41(10), 1931-1951. https://doi.org/1 $0.1080 / 0042098042000256323$ 
Ritchie, B. \& Beliveau, D. (1974). Hallmark events: An evaluation of a strategic response to seasonality in the travel market. Journal of Travel Research, 13(2), 14-20. https://doi. org/10.1177/004728757401300202

Robertson, M., Ong, F., Lockstone-Binney, L. \& Ali-Knight, J. (2018). Critical event studies: issues and perspectives. Event Management, 22(6), 865-874. https://doi.org/10.3727/ $152599518 \times 15346132863193$

Saarinen, J. (2006). Traditions of sustainability in tourism studies. Annals of Tourism Research, 33(4), 1121-1140. https://doi.org/10.1016/j.annals.2006.06.007

Salazar, N. (2016). Introduction: exposing sports mega-events through a mobilities lens. En N. Salazar, C. Timmerman, J. Wets, L. Gama \& S. Van Den Broucke (Eds.), Mega-Event Mobilities: A critical analysis. Routledge.

Sharpley, R. \& Stone, P.R. (2012). Socio-cultural impacts of events. En J. Page \& J. Cornell (Eds.), The Routledge Handbook of Events (pp. 347-361). Routledge.

Sheller, M. (2017). From spatial turn to mobilities turn. Current Sociology, 65(4), 1-17. https:// doi.org/10.1177/0011392117697463

Sheller, M. \& Urry, J. (2006). The new mobilities paradigm. Environment and Planning, 38(2), 207-226. https://doi.org/10.1068/a37268

Shin, H. B. (2012). Unequal cities of spectacle and mega-events in China. City, 16(6), 728744. https://doi.org/10.1080/13604813.2012.734076

Simonicca, A. (1997). Antropología del Turismo. Strategie di ricerca e contesti etnografici. La Nuova Italia Scientifica.

Spracklen, K. \& Lamond, I. (2016). Critical event studies. Routledge.

Spracklen, K., Richter, A. \& Spracklen, B. (2013). The eventization of leisure and the strange death of alternative Leeds. City, 17(2), 164-178. https://doi.org/10.1080/13604813 .2013 .765120

Stubbs, F. (2019). The Top 15 cities in Europe. Travel + Leisure. https://www.travelandleisure. com/worlds-best/cities-in-europe

Van Aalst, I. \& van Melik, R. (2012). City festivals and urban development: does place matter? European Urban and Regional Studies, 19(2), 195-206. https://doi.org/10.1177 10969776411428746

vv.AA. (2018). Best in Travel 2018: las 10 mejores ciudades. Lonely Planet. https://www. lonelyplanet.es/blog/best-in-travel-2018-las-10-mejores-ciudades

World Travel Tourism Council (wTTC). (2018). The Global Summit 2018. Buenos Aires Argentina. https://www.wttc.org/events/summits/previous-summits/the-global-summit-2018/ 